\title{
Observational clinical study in juvenile-adult glycogenosis type 2 patients undergoing enzyme replacement therapy for up to 4 years
}

\author{
C. Angelini • C. Semplicini $\cdot$ S. Ravaglia $\cdot$ B. Bembi $\cdot$ S. Servidei $\cdot$ E. Pegoraro $\cdot$ M. Moggio $\cdot$

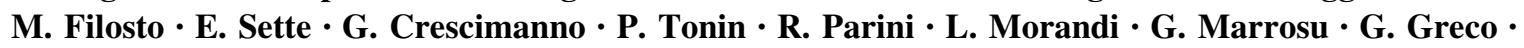 \\ O. Musumeci - G. Di Iorio - G. Siciliano · M. A. Donati · F. Carubbi · M. Ermani - T. Mongini • \\ A. Toscano $\cdot$ The Italian GSDII Group
}

Received: 19 August 2011/Revised: 7 October 2011/Accepted: 12 October 2011/Published online: 12 November 2011

(c) Springer-Verlag 2011

\begin{abstract}
The objective of this study was to describe a large Italian cohort of patients with late-onset glycogen storage disease type 2 (GSDII) at various stages of disease progression and to evaluate the clinical effectiveness
\end{abstract}

C. Angelini, T. Mongini, A. Toscano: Coordinators of the Italian Group on GSDII.

The members of the Italian GSDII Group are listed in the Appendix.

Electronic supplementary material The online version of this article (doi:10.1007/s00415-011-6293-5) contains supplementary material, which is available to authorized users.

C. Angelini $(\varangle) \cdot$ C. Semplicini · E. Pegoraro - M. Ermani Department of Neurosciences, University of Padova,

Via Giustiniani 5, 35128 Padova, Italy

e-mail: corrado.angelini@unipd.it

C. Angelini

IRCCS S. Camillo, Venice, Italy

S. Ravaglia

Department of Neurological Sciences, University of Pavia,

Pavia, Italy

S. Ravaglia

Clinical Institute "Beato Matteo", Vigevano, Italy

B. Bembi

Regional Coordination Centre for Rare Diseases, University

Hospital S. Maria Della Misericordia, Udine, Italy

S. Servidei

Department of Neurology, Università Cattolica, Rome, Italy

M. Moggio

Department of Neurology, Fondazione IRCCS Ca' Granda Osp. Policlinico, Centro Dino Ferrari, University of Milan, Milan, Italy of alglucosidase alpha enzyme replacement therapy (ERT). Previous studies showed in late-onset patients ERT efficacy against placebo and variable response in uncontrolled studies. Seventy-four juvenile or adult GSDII patients were treated with ERT in a multicenter open label, non-randomized study, from 12 months up to 54 months. Recombinant human alpha glucosidase (rh-GAA) was injected by intravenous route at $20 \mathrm{mg} / \mathrm{kg}$ every second week. Patients were divided into three groups according to ERT duration: Group A received treatment for 12-23 months $(n=16)$, Group B for 24-35 months $(n=14)$, and Group $\mathrm{C}$ for more than

M. Filosto

Clinical Neurology, Section for Neuromuscular Diseases and

Neuropathies, University Hospital Spedali Civili, Brescia, Italy

E. Sette

Neurology Unit, S. Anna Hospital, Ferrara, Italy

G. Crescimanno

Italian National Research Council, Institute of Biomedicine and Molecular Immunology, Palermo, Italy

P. Tonin

Department of Neurological Sciences and Vision,

Section of Neurology, University of Verona, Verona, Italy

R. Parini

Rare Metabolic Diseases Unit, Department of Pediatrics,

San Gerardo Hospital, Monza, Italy

L. Morandi

Immunology and Muscular Pathology Unit, National

Neurological Institute Carlo Besta, Milan, Italy

G. Marrosu

Neuromuscular Unit, Department of Cardiovascular Science and Neurology, University of Cagliari, Sardinia, Italy 
36 months $(n=44)$. Clinical assessment included a 6-min walk test (6MWT), forced vital capacity (FVC), the Walton and Gardner-Medwin score, the number of hours of ventilation, body mass index, echocardiography and blood creatine kinase (CK). Included in our cohort were 33 males and 41 females $(\mathrm{M}: \mathrm{F}=0.8: 1)$, with a mean age at first symptoms of 28.3 years (range 2-55 years) and a mean age of 43 years at study entry (range 7-72 years). Seven wheelchair bound patients, as well as 27 patients requiring ventilation support, were included. After treatment we could observe an increase in distance walked on the 6MWT in the large majority of patients $(48 / 58 ; 83 \%)$, with an overall mean increase of $63 \mathrm{~m}$ (from $320 \pm 161$ to $383 \pm 178 \mathrm{~m}$ ). After treatment in the majority of patients FVC was improved or unchanged $(45 / 69 ; 65 \%)$. In ventilated patients we observed an improvement in average number of hours off the ventilator (from 15.6 to $12.1 \mathrm{~h})$. Six patients stopped mechanical ventilation and two others started it. The effect of therapy was not related to ERT duration. Nine of 64 patients (13\%) that underwent to echocardiography showed a variable degree of cardiac hypertrophy (left ventriculum or septum), and a positive effect was observed after 36 months of ERT in one adult case. Discontinuation of treatment occurred in four patients: one drop-off case, one patient died for a sepsis after 34 months of treatment and two patients stopped ERT for worsening of general clinical condition. Mild adverse effects were observed in four cases $(5 \%)$. This study represents the largest cohort of late-onset GSDII patients treated with ERT, and confirm a positive

G. Greco

Department of Neurosciences, University of Siena, Siena, Italy

O. Musumeci · A. Toscano

Department of Neurosciences, Psychiatry and Anaesthesiology, University of Messina, Messina, Italy

G. Di Iorio

Department of Neurological Sciences, Second University of Naples, Naples, Italy

G. Siciliano

Neurological Institute, University of Pisa, Pisa, Italy

M. A. Donati

Clinic of Pediatric Neurology, Meyer Hospital, Florence, Italy

F. Carubbi

Department of Medicine, Endocrinology, Metabolism and Geriatrics, University of Modena and Reggio Emilia, Modena, Italy

T. Mongini

Centre for Neuromuscular Diseases "P. Peirolo", Department

of Neurosciences, University of Torino, Turin, Italy effect of treatment. These results, obtained in a large case series on therapy, indicate a favourable effect of ERT therapy, even in more advanced stage of the disease.

Keywords Glycogenosis type 2 - Enzyme replacement therapy · Acid maltase $\cdot$ Clinical trials

\section{Introduction}

Glycogenosis type II (GSDII) is an autosomal recessive disorder caused by the deficiency of the lysosomal enzyme acid $\alpha$-glucosidase (GAA) or acid maltase, which catalyses the hydrolysis of $\alpha-1,4$ and $\alpha-1,6$ links of glycogen $[1,2]$. The enzyme deficiency leads to lysosomal accumulation of glycogen, resulting in a spectrum of different clinical phenotypes, named according to age at onset in classical-infantile form, non-classical infantile form, childhood form, juvenile form and adult form. The last three forms are also known as late-onset Pompe disease. Many patients with adult-onset GSDII refer to minor symptoms beginning even in childhood, making age of onset unreliable to distinguish different phenotypes [3].

The rate of progression of the disease is faster when the onset of symptoms is earlier [1, 3]. Late-onset Pompe disease is characterized by progressive proximal muscle weakness and a variable degree of respiratory insufficiency. In untreated late onset patients, muscle strength and pulmonary function usually deteriorate, leading in a large majority of patients to wheelchair and need of respiratory support [4, 5]. Cardiac involvement, characterized by decreased left ventricular systolic function or elevated left ventricular or septum mass on echocardiogram, has been recently reported in few adult patients [6].

Supportive treatments, such as physical activity and special dietary regimes have been demonstrated to improve quality of life and motor function of GSDII patients, and may even delay disease progression in complicated patients [7]. In 2006, enzyme replacement therapy (ERT) using recombinant human acid alpha glucosidase (rh-GAA) was introduced and received broad-label market approval in Europe, and later in the US. Few studies on efficacy of treatment in late-onset patients have been published so far [8-12], demonstrating positive but variable effect of therapy in prolonged periods. Only one study included a placebo-controlled group [10].

The purpose of our study was to evaluate safety of ERT and its efficacy on motor, respiratory and cardiac function over the course of long-term treatment in a large cohort of Italian late-onset GSDII patients. 


\section{Materials and methods}

\section{Study design}

This study was designed as a spontaneous, non-sponsored open-label observational study and was conducted according to the principles of the Declaration of Helsinki on patient rights. The evaluation protocol was established in 2006 and adopted by 19 collaborating Italian Neuromuscular Centers (Padova, Ferrara, Verona, Brescia, two centers in Milano, Monza, Pavia, Torino, Siena, Roma, Napoli, Firenze, Pisa, Messina, Cagliari, Palermo, Udine and Modena) with no input or financial support from Genzyme Corporation (Cambridge, MA, USA), the manufacturer of Myozyme (rh-GAA). A compact disk with the standardized clinical examination protocols was made available to all participating institutions to increase intercentre reliability.

Patients were selected by three inclusion criteria: (1) confirmed diagnosis of GSDII, either by reduced GAA activity, below $35 \%$ of controls in skeletal muscle, leukocytes or fibroblasts, or by confirmed pathogenetic mutations in $G A A$ gene; (2) age at disease onset $\geq 2$ years; (3) presence of signs or symptoms of the disease (e.g. at least grade 1 on the Walton scale). After signing an informed consent, patients were enrolled, examined, and treated at the participating sites and included in the "Italian GAA ERT database". Data were sent to the coordinating centre at the University of Padova for statistical analysis. For the purpose of this study were considered only patients with treatment duration of more than 12 months.

\section{Clinical and instrumental examinations}

We obtained from each patient pre-treatment demographic and clinical history data, including gender, age at onset, age and disease duration at first infusion, use of ambulation devices, need for respiratory support, and co-morbidities (Supplemental Table 1). To be comparable with previously reported data, the 6-min walk test (6MWT) for motor function and forced vital capacity expressed as percentage of predicted value (FVC \%) for respiratory function were chosen as principal outcome measures. Global motor disability measured with the Walton and Gardner-Medwin Scale, cardiac function measured with echocardiography, BMI and blood tests for CK levels were also considered. All the tests were evaluated at baseline and every 6 months.

The 6MWT was performed to evaluate functional endurance during prolonged ambulation. It was performed as a standard walk in a 25-m long hospital corridor, and the distance walked was recorded in meters. FVC in the upright position, expressed as a percentage of the predicted value, was used as the major parameter for the evaluation of respiratory function. The Walton and Gardner-Medwin (WGM) Scale was used as a measure of global motor disability. Echocardiography was used to assess cardiac function. Not all patients could perform every test because of degree of motor or respiratory involvement, thus the numerosity of each sample is provided for each task.

In order to correct for ERT duration, patients were divided into three groups: Group A received treatment for 12-23 months, Group B for 24-35 months and Group C for over 36 months.

To evaluate the effect of molecular status on the response to ERT, GAA mutations were classified in splicing, missense and nonsense. Patients were grouped into nine categories according to the combination of mutation they presented; each category with a significant numerosity was considered.

\section{Adverse reactions}

The adverse reactions and tolerability of drug were investigated during the observation period by direct questioning.

\section{Statistical analysis}

Descriptive statistics (mean, range, standard deviation) were used to describe patient's demographic and clinical characteristics for continuous variables. Percentages were calculated for categorical variables. The Shapiro-Wilk test was used to check for normality of the data. For normally distributed variables, repeated measures analysis of variance was performed for testing the significance of the main effect of therapy (pre- vs. post-measures) and the first order interaction between therapy and sex, duration of therapy group and molecular status. For non-normal data, the Wilcoxon test for paired data and the Mann-Whitney $U$ test for unpaired data were used. The linear correlation between two variables was assessed by Spearman's Rho. The statistical significance was set at $p<0.05$.

\section{Results}

\section{Clinical characteristics}

From the Italian GAA ERT database 74 patients were selected based on the inclusion criteria (Supplemental Table 1). Included in our cohort were 33 males and 41 females $(\mathrm{M}: \mathrm{F}=0.8: 1)$. Mean age at first symptoms was 28.3 years $( \pm 15$; range $2-55$ years) and mean age at study entry was 43 years ( \pm 15.4 ; range: $7-72$ years). Average disease duration at first infusion was 14.7 years $( \pm 8.7$, range $1-35$ years). 
At baseline, 52 patients (70\%) could walk without support, six (8\%) could walk with unilateral support, nine $(12 \%)$ required bilateral support, and seven $(10 \%)$ were wheelchair bound. At study entry 47 patients $(64 \%)$ did not require ventilatory support, 12 patients $(16 \%)$ required ventilation support for less than $12 \mathrm{~h}$, and 15 (20\%) required a ventilator for more than $12 \mathrm{~h}$ per day.

GAA enzyme activity and molecular analysis

Both mutated GAA alleles were identified in 59 patients $(80 \%)$, only one mutated allele in 12 patients $(16 \%)$. In three patients (4\%) no mutations were identified. IVS1-13T $>\mathrm{G}$ was found in 62 cases $(62 / 74 ; 84 \%)$, always in heterozygous state. Other recurrent heterozygous mutations were the 525delT $(8 / 74 ; 11 \%)$, c. $2237 \mathrm{G}>\mathrm{A}(8 / 74 ; 11 \%)$, deletion of exon $18(6 / 74 ; 8 \%)$, c.1927 G>A (4/74; 5\%) and c.1465 $\mathrm{G}>\mathrm{A}(3 / 74 ; 4 \%)$. The IVS1-13T $>\mathrm{G}$ was present in $42 \%$ of mutated alleles (62/148) (Supplemental Table 1).

Residual enzymatic activity was measured in 58 patients in different tissues and resulted below normal limits. Due to different methods used in different laboratories has not been possible to obtain mean values.

\section{Motor function}

\section{Six minute walk test (6MWT)}

The 6MWT was available for 58 patients. The mean distance walked in the 6MWT at baseline and at the end of ERT study period increased from $320 \pm 161 \mathrm{~m}$ to $383 \pm 178 \mathrm{~m}(p<0.0001)$.

In order to verify if therapy duration was related to better performance, patients were clustered into three groups as previously described (Group A, $n=15$ patients; Group B, $n=9$ patients; Group C, $n=34$ ). The distance walked at the last evaluation increased significantly compared to baseline in all treatment groups. The changes were not correlated to therapy duration (interaction effect group and therapy, $p=0,91$; Fig. 1a). No effect of gender was found in the result of 6MWT (interaction effect sex and therapy, $p=0.90$ ). No linear correlations were found between the 6MWT change and the age at onset, the age at first infusion, or the disease duration (Spearman's Rho, $p>0.15$ ).

Forty-eight patients $(83 \%)$ increased and 10 patients (17\%) decreased the distance walked in the 6MWT on the final test (range 11, 312 vs. $-1,-78 \mathrm{~m}$ ). These two groups of patients differed significantly for mean disease duration at first infusion $(13.3 \pm 8.8 \mathrm{~min}$ ameliorated patients vs. $21.2 \pm 8.3 \mathrm{~m}$, Mann-Whitney $U$ test, $p=0.02$ ).

Fifty-four patients carried the leaky splicing IVS1-13T $>$ G mutation; these patients were divided
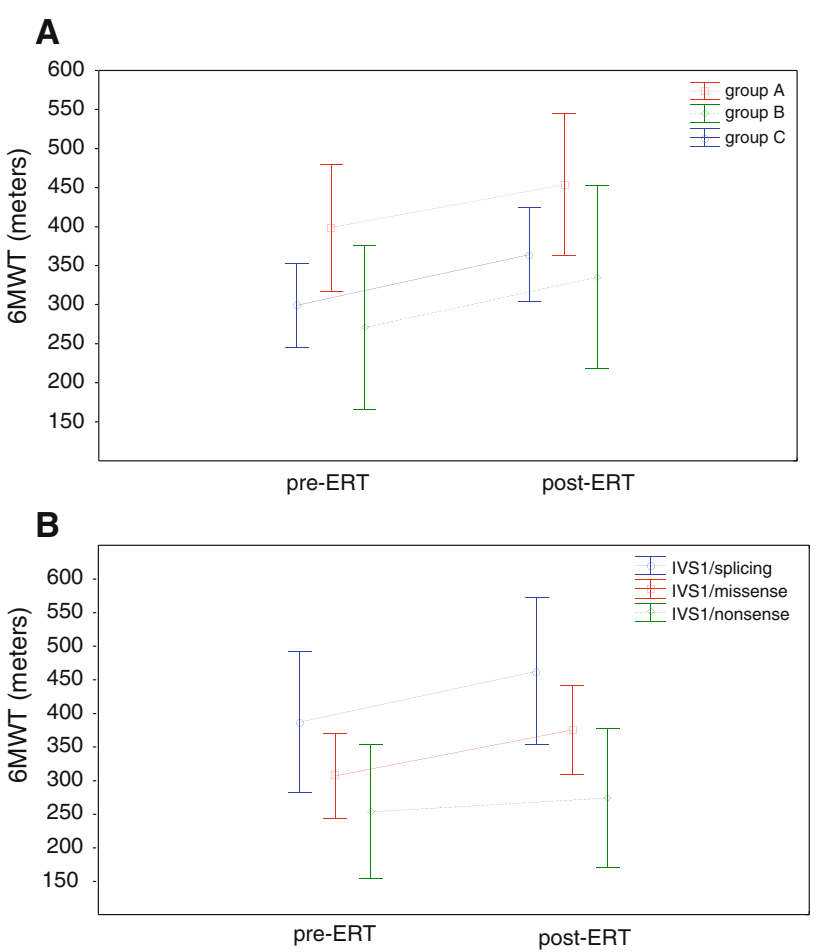

Fig. 1 a Six Minute Walk test, difference between pre- and post-ERT (meters). Group A: ERT duration 12-23 months, $n=15$. Group B: ERT duration 24-35 months, $n=9$. Group C: ERT duration 36 months or more, $n=34$. b Six Minute Walk test, difference between pre- and post-ERT (meters). Patients grouped according to the combination of mutation they presented: IVS1/splicing mutation, $n=9$; IVS1/missense mutation, $n=32$; IVS1/nonsense mutation, $n=13$

according to the type of mutation in the second allele in Group A: IVS1-13T $>$ G/splicing mutation, $n=9$; Group B: IVS1-13T $>$ G/missense mutation, $n=32$; Group C IVS1-13T $>$ G/nonsense mutation, $n=13$. The repeated measures ANOVA did not show significant effect of $G A A$ gene mutation (interaction effect of type of mutation and therapy, $p=0.24)$. Patients compound heterozygous IVS1 and splicing or missense mutations performed better compared to patients with nonsense mutations (Fig. 1b).

\section{Walton and Gardner-Medwin scale}

Global motor function was assessed using the WGM disability scale in 68 patients and it did not change significantly after treatment (Wilcoxon test, $p=0.22$ ). Eighteen patients $(18 / 68 ; 26 \%)$ improved and eight $(12 \%)$ had worsened motor function, 42 patients $(62 \%)$ remained stable. No difference was found in age at onset, disease duration or age at first infusion between the ameliorated/ unchanged patients compared to worsened patients (MannWithney $U$ test, $p=0.41, p=0.42$ and $p=0.92$ respectively). 


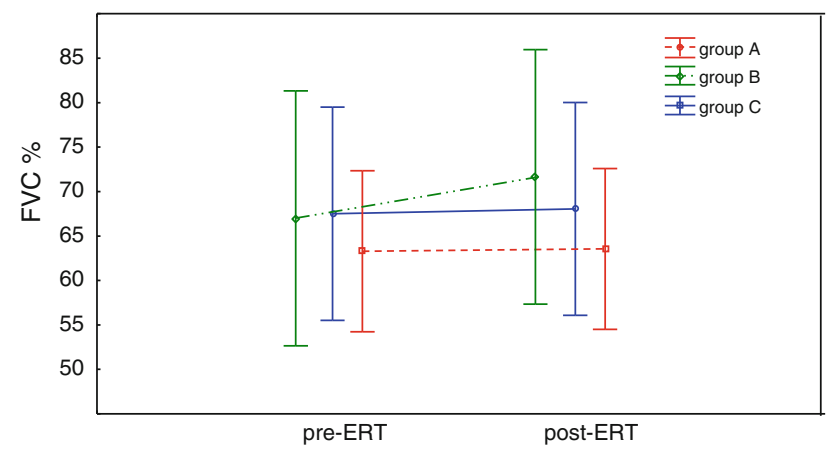

Fig. 2 Forced Vital Capacity (FVC) as percentage of predicted value, difference between pre- and post-ERT. Group A: ERT duration 12-23 months, $n=20$. Group B: ERT duration $24-35$ months, $n=14$. Group C: ERT duration 36 months or more, $n=35$

\section{Respiratory function}

Standard spirometry was performed in the upright position before and after ERT in 69 patients. At baseline mean $\mathrm{FVC} \%$ was $65.2 \pm 26.5$, ranging from 14 to 138 , and after treatment it was $66.5 \pm 26.6(p=0.22)$. There were no significant changes in $\mathrm{FVC} \%$ compared to baseline in any treatment group (Fig 2a).

In 45 patients $(65 \%)$ we observed an increase or stabilization of $\mathrm{FVC} \%$ and in 24 patients $(35 \%)$ a reduction. Between the ameliorated/unchanged patients compared to worsened patients no differences in disease duration, age at onset and age at first infusion were observed (MannWithney $U$ test, $p=0.22, p=0.50$ and $p=0.99$, respectively).

Six patients stopped the ventilatory support during the treatment period, while two patients started non-invasive ventilation. Furthermore, the 21 patients that continued to need ventilatory support showed a significant reduction of number of hours/day in ventilation (mean from 15.6 to $12.1 \mathrm{~h}, p=0.005$ ) (Fig. 2b).

\section{Cardiac function}

Nine of the 64 patients (14\%) studied with echocardiography showed a variable degree of left ventricular and/or septal hypertrophy (one juvenile, eight adult cases), their ages ranging from 18 to 66 years. In three of them a history of hypertension was known. After 36 months of ERT in one patient cardiac hypertrophy disappeared and his follow-up cardiac echocardiography was within normal limits; no significant changes were evidenced in the other cases.

\section{Muscle enzymes}

CK level tended to decrease during ERT in $76 \%$ patients; in $17 \% \mathrm{CK}$ level increased and 7\% CK showed no changes.
No significant correlations with other factors, such as age, therapy duration or physical activity, were found.

Body mass index

Body mass index was available for 47 patients. No significant changes between baseline and after ERT were seen in 47 patients, from $24.23 \pm 5.3$ to $24.56 \pm 5.3$, $p=0.10$. Stratifying patients according to BMI at baseline in underweight $(\mathrm{BMI}<18.5, n=6)$, normal weight (BMI $=18.5-24.9, \quad n=22) \quad$ and overweight-obesity (BMI $>25, n=19$ ) no significant changes were found; however, in underweight patients an increased BMI after treatment was detected (from $15.48 \pm 1.90$ to $16.9 \pm 2$, $p=0.10)$.

\section{Safety of therapy}

Discontinuation of treatment occurred in four patients, one drop-off case and two patients that worsened their general clinical condition and decided not to continue. One patient died after 34 months of treatment from sepsis. Mild adverse effects were observed in four patients $(6 \%)$ and were characterized by facial or infusion site erythema, flulike syndrome, generalized itch or bronchospasm. Premedication with antihistamines (e.g., cetirizine or chlorphenamine) and corticosteroids (e.g., metilprednisolone $40 \mathrm{mg}$ ) allowed continuation of ERT in every case.

Antibody titer values were available for 15 patients, resulting negative in four cases and elevated in 11 (ranging from 1:800 to $1: 102,400)$. The patient with the highest antibody titer presented flu-like reaction during every infusion, and was successfully treated with premedication. No further correlations between antibody titer and infusions reactions were observed.

\section{Discussion}

In 2006, enzyme replacement therapy with rh-GAA received marketing approval on the basis of clinical trials for classic infantile GSDII. Only a limited number of ERT trials [8-12] have been published on late-onset phenotypes. The most numerous series of late onset GSDII cases is the LOTS study where 90 patients between 10 and 70 years of age were treated with rh-GAA (60 patients) or placebo (30 cases), for an 18 month period [10]. In the multicenter observational study published by Strothotte 44 late-onset GSDII cases (21-69 years old) were treated for 12 months [9]. These studies demonstrated clinical efficacy with ERT treatment in prolonging distance walked and stabilizing or slightly improving respiratory function, measured with forced vital capacity and inspiratory and expiratory 
pressures. At the same time, they pointed out the difficulties to obtain consistent outcome measures in late-onset GSDII patients due to the heterogeneous presentation of the disease and natural course and the high variability of individual response.

Our study is extended for the longest observational period so far done and confirms the major findings of these two previous series published. However, it addresses also additional relevant points: safety of ERT on long observation period and effect of ERT on severely affected patients. While the LOTS study [10] included only walking and non-ventilated patients, we also include severely affected patients that required a ventilator $(36 \%)$ or were confined to wheelchairs (10\%). This more severely afflicted cohort necessitated careful clinical assessment measures. We observed that six patients discontinued ventilation and in 22 case significantly reduced the number of hours/day in ventilation.

One limitation of our study is the absence of a control non-treated group. The clinical progression can be extrapolated from natural history studies and LOTS study $[4,5,10]$, which generally demonstrates a relentless deterioration in both motor and respiratory function.

In our independent study we observed improvement of motor function, consistently with the LOTS study results, which persisted in time as demonstrated by the group of patients treated for over 36 month. Moreover, we demonstrated positive effects on respiratory function in terms of hours of ventilation in more severely affected patients. Safety and efficacy were observed in smaller series of patients [8-12]; our large follow up study confirms and extends these previous observations on late-onset GSDII cases, studied for longer periods.

In our series the cardiac hypertrophy was seen in $14 \%$ of juvenile-adult cases, not reported in previous studies, and this stresses the importance of regular cardiac evaluation, even in adult patients. In one case we also documented that ERT did improve heart condition.

Every clinician could observe, in this case series such as in previous studies, that some patients respond better to enzyme replacement therapy [13]. One outcome measure seems not to be sufficient to subgroup patients in responder or not responder, as some ameliorate the result of only few tests, indicating a different pattern of response in motor and respiratory or cardiac function. Despite the high numerosity of this patients' population, few significant correlations were found between patients' clinical and demographic characteristics, molecular mutations or clinical status and the changes of functions after treatment. Shorter disease duration and milder mutation seem to be the best predictors of good response to ERT, but more studies are needed.

Our observations suggest that ERT mostly enhanced the muscle endurance, given the 6MWT results. This might be due to a different therapy response of different muscle fiber types and connective tissue involvement, to a different body composition and volume of distribution of enzymes, or to a variable mannose receptor density in different muscles. Enzyme replacement may also antagonize muscle fiber autophagy, but only future morphological and functional observations from control and post-ERT muscle biopsies, can determine the cause of improved motor performance induced by long-term ERT.

This study represents the largest cohort of late-onset GSDII patients undergoing to ERT for a long period, and further supports a positive effect of treatment in GSDII late-onset patients.

Conflict of interest Drs Toscano and Angelini are members of the Pompe Global Advisory Board, sustained by Genzyme, and received reimbursement for the participation to the board meetings. Drs Angelini, Toscano, Bembi, Servidei, Filosto and Semplicini received support from Genzyme for organizing or participating to scientific meetings.

\section{Appendix: The Italian GSDII Group}

L. Vercelli, Centre for Neuromuscular Diseases "P. Peirolo", Department of Neurosciences, University of Torino, Torino, Italy; R. di Giacopo, Department of Neurology, Università Cattolica, Rome, Italy; V. Lucchini and G. P. Comi, Fondazione IRCCS Ca' Granda Osp. Policlinico, Dept. of Neurology, Centro Dino Ferrari, University of Milan, Italy; V. Tugnoli, Neurology Unit, S. Anna Hospital, Ferrara, Italy; M. Rigoldi, Rare Metabolic Diseases Unit, Department of Pediatrics, San Gerardo Hospital, Monza, Italy; M. B. Pasanisi, Immunology and Muscular Pathology Unit, National Neurological Institute Carlo Besta, Milan, Italy; R. Piras, Neuromuscular Unit, Department of Cardiovascular Science and Neurology, University of Cagliari, Italy; F. Giannini, Departments of Neurosciences, University of Siena, Italy; E. Barca, Department of Neurosciences, Psychiatry and Anaesthesiology, University of Messina, Messina, Italy; S. Gasperini, Clinic of Pediatric Neurology, Meyer Hospital, Firenze, Italy; G. Ricci, Neurological Institute, University of Pisa, Pisa, Italy; D. Diodato, Department of Neurological Sciences, Second University of Naples, Italy; A. Ariatti, Department of Medicine, Endocrinology, Metabolism and Geriatrics, University of Modena and Reggio Emilia, Modena, Italy.

\section{References}

1. Hirschhorn R, Reuser AJ (2001) Glycogen storage disease type II: acid alphaglucosidase (acid maltase) deficiency. In: Scriver CR, Sly W, Valle D (eds) The metabolic and molecular bases of 
inherited disease, 8th edn edn. McGraw-Hill, New York, pp 3389-3420

2. Engel AG, Hirschhorn R (1994) Acid maltase deficiency. In: Engel A, Franzini-Armstrong C (eds) Myology: basic and clinical. McGraw-Hill, New York, pp 1533-1553

3. Laforet P, Nicolino M, Eymard PB et al (2000) Juvenile and adultonset acid maltase deficiency in France: genotype-phenotype correlation. Neurology 55:1122-1128

4. Hagemans ML, Winkel LP, Van Doorn PA, Hop WJ, Loonen MC, Reuser AJ, Van der Ploeg AT (2005) Clinical manifestation and naturai course of late-onset Pompe's disease in 54 Dutch patients. Brain 128(Pt 3):671-677

5. Winkel LP, Hagemans ML, van Doorn PA, Loonen MC, Hop WJ, Reuser AJ, van der Ploeg AT (2005) The natural course of nonclassic Pompe's disease: a review of 225 published cases. J Neurol 252:875-884

6. Forsha D, Li JS, Smith PB, van der Ploeg AT, Kishnani P, Pasquali SK (2011) Late-onset treatment study investigators cardiovascular abnormalities in late-onset Pompe disease and response to enzyme replacement therapy. Genet Med 13:625-631

7. Slonim AE, Bulone L, Goldberg T, Minikes J, Slonim E, Galanko J, Martiniuk F (2007) Modification of the naturai history of adultonset acid maltase deficiency by nutrition and exercise therapy. Muscle Nerve 35(1):70-77

8. van Capelle CI, Winkel LP, Hagemans ML, Shapira SK, Arts WF, van Doorn PA, Hop WC, Reuser AJ, van der Ploeg AT (2008) Eight years experience with enzyme replacement therapy in two children and one adult with Pompe disease. Neuromuscul Disord 18(6):447-452
9. Strothotte S, Strigi-Pill N, Grunert B, Kornblum C, Eger K, Wessig C, Deschauer M, Breunig F, Glocker FX, Vielhaber S, Brejova A, Hilz M, Reiners K, Muller-Felber W, Mengel E, Spranger M, Schoser B (2010) Enzyme replacement therapy with alglucosidase alfa in 44 patients with late-onset glycogen storage disease type 2: 12-month results of an observational clinical trial. J Neurol 257:91-97

10. van der Ploeg AT, Clemens PR, Corzo D, Escolar DM, Florence J, Groeneveld GJ, Herson S, Kishnani PS, Laforet P, Lake SL, Lange DJ, Leshner RT, Mayhew JE, Morgan C, Nozaki K, Park DJ, Pestronk A, Rosenbloom B, Skrinar A, van Capelle CI, van der Beek NA, Wasserstein M, Zivkovic SA (2010) A randomized study of alglucosidase alfa in late-onset Pompe's disease. N Engl J Med 362(15):1396-1406

11. Bembi B, Pisa FÉ, Gonfalonieri M, Ciana G, Fiumara A, Parini R, Rigoldi M, Moglia A, Costa A, Carlucci A, Danesino C, Pittis MG, Dardis A, Ravaglia S (2010) Long-term observational, nonrandomized study of enzyme replacement therapy in late-onset glycogenosis type II. J Inherit Metab Dis 33:727-735

12. van Capelle CI, van der Beek NA, Hagemans ML, Arts WF, Hop WC, Lee P, Jaeken J, Frohn-Mulder IM, Merkus PJ, Corzo D, Puga AC, Reuser AJ, van der Ploeg AT (2010) Effect of enzyme therapy in juvenile patients with Pompe disease: a three-year open-label study. Neuromusc Disord 20:775-782

13. van der Ploeg AT (2010) Where do we stand in enzyme replacement therapy in Pompe's disease? Neuromusc Disord 20:773-774 